\title{
The Ionization Energies of Dust-Forming Metal Oxide Clusters
}

\author{
David Gobrecht ${ }^{1, *}{ }^{\mathbb{D}}$, Jan Philip Sindel ${ }^{1,2,3}$, Helena Lecoq-Molinos ${ }^{1}$ and Leen Decin ${ }^{1}$ \\ 1 Institute of Astronomy, KU Leuven, Celestijnenlaan 200D, 3001 Leuven, Belgium; \\ jps23@st-andrews.ac.uk (J.P.S.); helena.lecoqmolinos@student.kuleuven.be (H.L.-M.); \\ leen.decin@kuleuven.be (L.D.) \\ 2 SUPA, School of Physics \& Astronomy, University of St Andrews, North Haugh, St Andrews KY169SS, UK \\ 3 Centre for Exoplanet Science, University of St Andrews, North Haugh, St Andrews KY169SS, UK \\ * Correspondence: dave@gobrecht.ch
}

Citation: Gobrecht, D.; Sindel, J.P.; Lecoq-Molinos, H.; Decin, L. The Ionization Energies of Dust-Forming Metal Oxide Clusters. Universe 2021, 7, 243. https://doi.org/10.3390/ universe7070243

Academic Editors: Sergio Cristallo and Paolo Ventura

Received: 15 March 2021

Accepted: 10 July 2021

Published: 14 July 2021

Publisher's Note: MDPI stays neutral with regard to jurisdictional claims in published maps and institutional affiliations.

Copyright: (c) 2021 by the authors. Licensee MDPI, Basel, Switzerland. This article is an open access article distributed under the terms and conditions of the Creative Commons Attribution (CC BY) license (https:// creativecommons.org/licenses/by/ $4.0 /)$.

\begin{abstract}
Stellar dust grains are predominantly composed of mineralic, anorganic material forming in the circumstellar envelopes of oxygen-rich AGB stars. However, the initial stage of the dust synthesis, or its nucleation, is not well understood. In particular, the chemical nature of the nucleating species, represented by molecular clusters, is uncertain. We investigated the vertical and adiabatic ionization energies of four different metal-oxide clusters by means of density functional theory. They included clusters of magnesia $(\mathrm{MgO})_{n}$, silicon monoxide $(\mathrm{SiO})_{n}$, alumina $\left(\mathrm{Al}_{2} \mathrm{O}_{3}\right)_{n}$, and titania $\left(\mathrm{TiO}_{2}\right)_{n}$ with stoichiometric sizes of $\mathrm{n}=1-8$. The magnesia, alumina, and titania clusters showed relatively little variation in their ionization energies with respect to the cluster size n: 7.1-8.2 eV for $(\mathrm{MgO})_{\mathrm{n}}$, from 8.9-10.0 eV for $\left(\mathrm{Al}_{2} \mathrm{O}_{3}\right)_{\mathrm{n}}$, and 9.3-10.5 eV for $\left(\mathrm{TiO}_{2}\right)_{\mathrm{n}}$. In contrast, the $(\mathrm{SiO})_{\mathrm{n}}$ ionization energies decrease with size $\mathrm{n}$, starting from $11.5 \mathrm{eV}$ for $\mathrm{n}=1$, and decreasing to $6.6 \mathrm{eV}$ for $\mathrm{n}=8$. Therefore, we set constraints on the stability limit for neutral metal-oxide clusters to persist ionization through radiation or high temperatures and for the nucleation to proceed via neutral-neutral reactions.
\end{abstract}

Keywords: metal oxide; ionization; clusters; condensation; cations; dust; silicates; circumstellar; minerals; bottom-up

\section{Introduction}

The formation of dust in asymptotic giant branch (AGB) star envelopes is not well understood [1]. In particular, the dust nucleation around oxygen-rich AGB stars is uncertain, since it is not clear which chemical species initiate and partake in the condensation process [2]. The composition of a dust grain, once it has formed and grown to a size on the (sub)micron scale, is known from the analysis of meteoritic stardust [3]. A great variety of different materials have been identified in presolar stardust grains, with silicon-oxide compounds, or silicates, being the major constituents [4,5]. The silicates are found to be magnesium-rich (rather than iron-rich) with a mixed olivine-pyroxene stoichiometry, of which only a fraction is crystalline [6-10].

The condensation temperatures of silicate material are known to be about 900-1300 K around AGB stars [11,12]. However, large circumstellar dust grains with sizes of $0.6 \mu \mathrm{m}$ form within two stellar radii, where the temperatures are higher [13]. Moreover, a dust formation zone close to the star is required to drive the mass loss of an AGB star [14]. Therefore, it is likely that silicates do not nucleate on their own (i.e., homogeneously), but heterogeneouslyon the surfaces of previously formed seed nuclei. The nature (composition, size, crystallinity) of these seed nuclei is still a matter of debate [15]. Owing to their minor contribution to the total dust mass, seed nuclei can also be made of less abundant elements. However, a crucial requirement for seed nuclei is to be refractory. Alumina and titania fulfill this criterion, and in addition, they are promising carriers of a spectral dust feature, commonly observed at a wavelength of $13 \mu \mathrm{m}$ in oxygen-rich AGB stars [16-18]. Albeit silicon monoxide and magnesia are less refractory and hence less likely to trigger dust 
nucleation in AGB stars, they represent basic building blocks of silicates and are included in our study. Previous studies have addressed the four nucleation candidates $\mathrm{MgO}, \mathrm{SiO}$, $\mathrm{Al}_{2} \mathrm{O}_{3}$, and $\mathrm{TiO}_{2}$, and described the properties of the related neutral clusters in astrophysical environments theoretically [19-21], and experimentally [22-24]. We note, however, that it is probable that chemically-heterogeneous compounds involving several clustering species could play a role in the rich gas-phase mixtures of AGB circumstellar envelopes.

Nucleation is commonly presumed to proceed via neutral-neutral reactions. An aspect that is often ignored in the studies of dust nucleation is the degree of ionization, or the fraction of matter that is charged. AGB stars are rather $\operatorname{cool}(T=2000-3000 \mathrm{~K})$, resulting in a stellar radiation field with a relatively mild UV contribution. However, pulsation-induced shocks propagate periodically through the AGB envelopes, enhancing the temperatures locally and temporarily [25]. In addition, some AGB stars reside in binary systems hosting a hot companion star providing a source of ionizing UV radiation, as for example Mira B. Moreover, interstellar UV radiation can have an ionizing effect on the gas, particularly at larger distances from the star [26]. Therefore, we aim to constrain the conditions under which oxygen-rich dust can nucleate through neutral metal-oxide clusters and find the temperatures at which their related cations become important.

This study represents a continuation of [15], which investigated the nucleation of neutral clusters of four metal oxide families $\left(\mathrm{MgO}, \mathrm{SiO}, \mathrm{Al}_{2} \mathrm{O}_{3}\right.$, and $\left.\mathrm{TiO}_{2}\right)$. Here, we address the corresponding ionization energies and their related cations allowing us to assess whether a fast ion-molecule chemistry can take place and compete with a comparatively slow neutral-neutral nucleation.

This paper is organized as follows. In Section 2, we describe the methods to calculate the ionization potentials. Section 3 addresses the results obtained for the four metal oxide clusters. Finally, we summarize and discuss our findings in Section 4.

\section{Methods}

Our study focuses on four different metal oxide nucleation candidates that were presented in [15]. They include clusters of $(\mathrm{MgO})_{n},(\mathrm{SiO})_{\mathrm{n}},\left(\mathrm{Al}_{2} \mathrm{O}_{3}\right)_{\mathrm{n}}$, and $\left(\mathrm{TiO}_{2}\right)_{\mathrm{n}}$ and sizes $\mathrm{n}=1-8$. We performed density functional theory (DFT) calculations of the neutral cluster and its singly ionized cation to compute the ionization potential, or ionization energy $E_{i}\left(X_{n}\right)$, for each species $X$ and each size $n$ :

$$
E_{i}\left(X_{n}\right)=E\left(X_{n}^{+}\right)-E\left(X_{n}\right)
$$

where $E\left(X_{n}^{+}\right)$and $E\left(X_{n}\right)$ are the potential energies of $X_{n}^{+}$and $X_{n}$, respectively. For reasons of comparability all DFT calculations were performed at the same level of theory. We chose the B3LYP/6-311+(d) hybrid density functional/basis set [27], as it provides reasonable accuracy at an affordable computational cost, and is suitable for inorganic metal oxides [28]. All DFT calculations were performed with the software suite of Gaussian 09 [29].

For our calculations, we assumed that the neutral metal oxide clusters are present in the form of their global minimum (GM) candidate structure. The corresponding geometries originated from [30] for $\mathrm{MgO}$, from [20] for $\mathrm{SiO}$, from [31,32] for $\mathrm{Al}_{2} \mathrm{O}_{3}$, and from [33] for $\mathrm{TiO}_{2}$, and were subsequently optimized. The optimization was performed for both the neutral cluster $X$ and its cation $X^{+}$, and included vibration frequency analysis. This analysis allowed us to discriminate between true minima with only real frequencies and transition states (TS) with an imaginary vibration mode.

Apart from the energy and the charge, the spin multiplicity of the cations was also different from their neutral counterparts. Here, all cations were presumed to be in doublet state (i.e., with a spin multiplicity of 2), since the neutral metal oxide clusters are singlet states, apart from the triplet $\mathrm{Al}_{2} \mathrm{O}_{3}$ monomer. Test calculations on small sized $(\mathrm{n}=1-4)$ metal-oxide cations in quartet states (i.e., with a spin multiplicity of 4$)$ showed higher potential energies than their corresponding doublet states (if they converged at all). We distinguished between vertical and adiabatic ionization energies. The vertical ionization energies are evaluated according to single point energies. In contrast, adiabatic 
ionization energies take into account structural rearrangements that are caused by the ionization process (i.e., by removing an electron) and are consequently lower than the vertical ionization energies.

\section{Results}

\subsection{Vertical Ionization}

In Table 1 the vertical (and adiabatic) ionization energies of the four oxide families (magnesia, silicon monoxide, alumina, titania) are listed as a function of cluster size $n$.

Table 1. The vertical and adiabatic ionization energies, $\mathrm{E}^{\mathrm{v}}$ and $\mathrm{E}^{\mathrm{a}}$, given in units of $\mathrm{eV}$ for the four considered metal oxide clusters $\mathrm{MgO}, \mathrm{SiO}, \mathrm{Al}_{2} \mathrm{O}_{3}$, and $\mathrm{TiO}_{2}$ as a function of cluster size $\mathrm{n}=1-8$.

\begin{tabular}{ccccccccc}
\hline & \multicolumn{2}{c}{$(\mathbf{M g O})_{\mathbf{n}}$} & \multicolumn{2}{c}{$(\mathbf{S i O})_{\mathbf{n}}$} & \multicolumn{2}{c}{$\left(\mathbf{A l}_{\mathbf{2}} \mathbf{O}_{\mathbf{3}} \mathbf{n}_{\mathbf{n}}\right.$} & \multicolumn{2}{c}{$\left(\mathbf{T i O}_{\mathbf{2}} \mathbf{n}_{\mathbf{n}}\right.$} \\
$\mathbf{n}$ & $\mathbf{E}^{\mathbf{v}}$ & $\mathbf{E}^{\mathbf{a}}$ & $\mathbf{E}^{\mathbf{v}}$ & $\mathbf{E}^{\mathbf{a}}$ & $\mathbf{E}^{\mathbf{v}}$ & $\mathbf{E}^{\mathbf{a}}$ & $\mathbf{E}^{\mathbf{v}}$ & $\mathbf{E}^{\mathbf{a}}$ \\
\hline 1 & 7.86 & 7.75 & 11.49 & 11.49 & 9.39 & 9.15 & 9.81 & 9.67 \\
2 & 7.82 & 7.56 & 9.22 & 9.21 & 9.81 & 9.52 & 10.50 & 10.23 \\
3 & 8.19 & 8.18 & 9.01 & 8.74 & 9.98 & 9.45 & 9.92 & 9.77 \\
4 & 7.90 & 7.37 & 8.39 & 8.34 & 9.88 & 9.52 & 10.54 & 10.43 \\
5 & 7.61 & 7.09 & 8.20 & 7.66 & 9.74 & 9.48 & 10.25 & 9.32 \\
6 & 7.94 & 7.63 & 7.89 & 7.30 & 9.73 & 9.54 & 10.32 & 9.34 \\
7 & 7.74 & 7.23 & 8.05 & 7.35 & 9.72 & 9.48 & 9.40 & 9.27 \\
8 & 7.54 & 7.16 & 7.04 & 6.62 & 9.13 & 8.90 & 10.08 & 9.04 \\
\hline
\end{tabular}

In Figure 1 the vertical and adiabatic ionization energies are represented graphically. Magnesia clusters show a narrow range of 7.54-7.94 eV in their vertical ionization energies, apart from the trimer $(\mathrm{n}=3)$, which is explained in Section 3.2. These values are lower than the experimental value of $8.76 \pm 0.22 \mathrm{eV}$ [34] for the $\mathrm{MgO}^{+}$cation. By inspecting the NIST Computational Chemistry Comparison and Benchmark Database (CCCBDB) database http:/ / cccbdb.nist.gov/, accessed on 1 March 2021, we found that the highlevel coupled cluster and the majority of the hybrid functionals underpredict the $\mathrm{MgO}$ ionization energies by about $1 \mathrm{eV}$. The deviation from the experimental result seems, therefore, to be inherent regarding DFT calculations. The largest ionization energy in our study $(11.49 \mathrm{eV})$ was found for the $\mathrm{SiO}$ molecule, which is in excellent agreement with the experimental results [35], which a value of $(11.49 \pm 0.02 \mathrm{eV})$. Larger-sized $(\mathrm{SiO})_{\mathrm{n}}, \mathrm{n}>1$, polymers, showed a decreasing trend with cluster size $n$, except for $n=7$. Clusters of $\mathrm{Al}_{2} \mathrm{O}_{3}$ showed vertical ionization energies in a narrow range of 9.39-9.98 eV, apart for the largest considered size $(n=8)$. To our knowledge there is no study that investigated $\mathrm{Al}_{2} \mathrm{O}_{3}{ }^{+}$ experimentally. However, we note that the calculated alumina cluster ionization energies closely resemble the experimental value of $9.46 \pm 0.06 \mathrm{eV}$ for the $\mathrm{AlO}$ molecule [36] and the value of $9.46 \mathrm{eV}$ for its dimer, $\mathrm{Al}_{2} \mathrm{O}_{2}$, calculated by [37]. With the exception of the previously mentioned $\mathrm{SiO}$ molecule, the $\mathrm{TiO}_{2}$ clusters exhibited the largest vertical ionization energies, ranging from 9.40 to $10.54 \mathrm{eV}$. As far as we know, there is no laboratory measurement of the $\mathrm{TiO}_{2}{ }^{+}$cation and its related ionization energy. However, the existence of the cation was proven experimentally [38]. With the exception of $(\mathrm{MgO})_{5}$, all four cluster families showed their lowest adiabatic ionization energy at their largest cluster size $(n=8)$. 


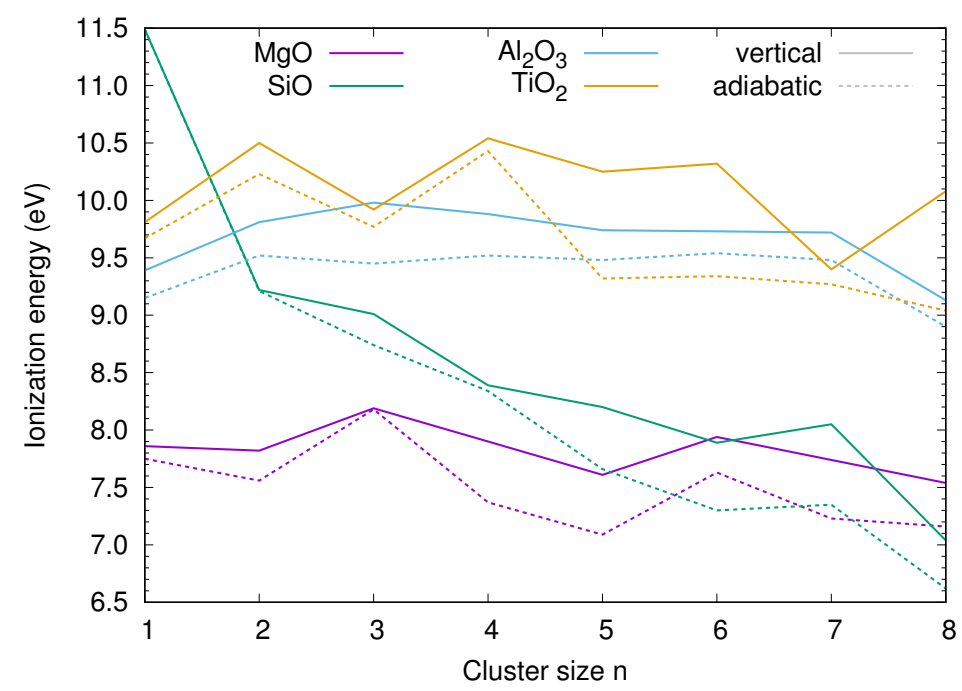

Figure 1. The vertical (straight lines) and adiabatic (dashed lines) ionization energies (in $\mathrm{eV}$ ) of the metal oxide clusters as a function of cluster size $n$.

\subsection{Adiabatic Ionization}

In the following, we present the structures of the adiabatically optimized cluster cations, compare them with the neutral clusters, and discuss their relative energies with respect to the vertical ionization energies.

\subsubsection{Magnesia Cations}

The adiabatically optimized $\mathrm{MgO}^{+}$cation has a larger bond distance $(\mathrm{d}=1.858 \AA)$ than the neutral $\mathrm{MgO}$ molecule $(1.767 \AA)$, as can be seen in Figure 2. The corresponding electron removal accounts for a difference of $0.09 \AA$ and $0.11 \mathrm{eV}$. Both neutral $(\mathrm{MgO})_{2}$ and its cation show flat square structures with equidistant edges. The cation has slightly larger bond lengths $(1.912 \AA)$ as compared to the neutral dimer (1.892 $\AA$ ). It is thus impracticable to localize the electron removal through the ionization process. The neutral $(\mathrm{MgO})_{3}$ cluster structure is a ring consisting of six equidistant $\mathrm{Mg}-\mathrm{O}$ bonds with a bond length of $1.852 \AA$. Our initial searches for $(\mathrm{MgO})_{3}{ }^{+}$resulted in convergence failures. However, we found a rectangular-shaped cyclic structure to be a suitable candidate for the cationic form of $(\mathrm{MgO})_{3}$. We note that a completely flat 2D geometry exhibits an imaginary vibration mode and is therefore likely to be a TS. A slight distortion of the geometry out of the 2D plane leads to a true minimum with only real vibration modes. The adapted shape of $(\mathrm{MgO})_{3}{ }^{+}$is arguably the reason for the highest adiabatic ionization energy among the $(\mathrm{MgO})_{\mathrm{n}}$ clusters. Neutral $(\mathrm{MgO})_{4}$ has the shape of a cube with identical edge (bond) lengths of $1.972 \AA$. In its ionized state, the $(\mathrm{MgO})_{4}{ }^{+}$bond lengths are altered in a non-uniform way, where diagonally opposed edges have the same lengths. The longest two distances (marked with a dashed line) have a length of $2.077 \AA$, which is likely to be the location of the electron removal. In neutral $(\mathrm{MgO})_{5}$ the bond lengths are not identical, but show a range from $1.82 \AA$ to 2.00 $\AA$. After removing an electron, the $(\mathrm{MgO})_{5}{ }^{+}$bond lengths change in the region of the two rhombic substructures. Most prominent are two distances exceeding $2 \AA$ (marked with a dashed line) indicating the location of the electron disposal. The geometry of neutral $(\mathrm{MgO})_{6}$ consists of two 'honeycomb' hexagons stacked on top of each other. The bond lengths within the two hexagons are $1.925 \AA$, and the hexagons are connected by relatively large distances $(2.014 \AA)$. The optimization of $(\mathrm{MgO})_{6}{ }^{+}$cation led to a TS. By distorting the TS geometry we found a real $(\mathrm{MgO})_{6}{ }^{+}$minimum showing two large $\mathrm{Mg}-\mathrm{O}$ bonds of $2.02 \AA$. Moreover, the hexagons in the cation are not plane-parallel as in the neutral $n=6$ cluster. For $n=7$, the bond lengths of the neutral cluster range from 1.90 to $1.97 \AA$. The largest effects on the cation geometry were found for two $\mathrm{Mg}-\mathrm{O}$ bonds with large bond lengths of $2.08 \AA$ (marked with dashed lines). The neutral $(\mathrm{MgO})_{8}$ cluster has a cylindrical 
shape with a square base (edge length $1.959 \AA$ ). The remaining bond lengths range from 1.94 to $1.97 \AA$. However, the cationic form $(\mathrm{MgO})_{8}{ }^{+}$did not converge, unless we distorted its geometry. The distorted geometry resembles the shape of a "keyhole", corresponding to the second-lowest energy neutral $(\mathrm{MgO})_{8}$ isomer. Therefore, we use the latter "keyhole" structure as a reference. Its adiabatic ionization impacts most strongly two $\mathrm{Mg}-\mathrm{O}$ bonds that exceed a distance of $2 \AA$ (marked with dashed lines).

For $\mathrm{n}=2$, the site of the ionization could not be attributed to a specific location. $(\mathrm{MgO})_{3}{ }^{+}$represents a special case, as its structure was re-adjusted, and thus its ionization energy represents an outlier. For $\mathrm{n}=4-8$, the adiabatic ionization increases two $\mathrm{Mg}-\mathrm{O}$ bonds simultaneously. Therefore, the ionization locations of the $(\mathrm{MgO})_{\mathrm{n}}, \mathrm{n}=2-8$, could not be uniquely determined. This might be the result of electron delocalization, but it could also arise from approximating the electron probability by its density.
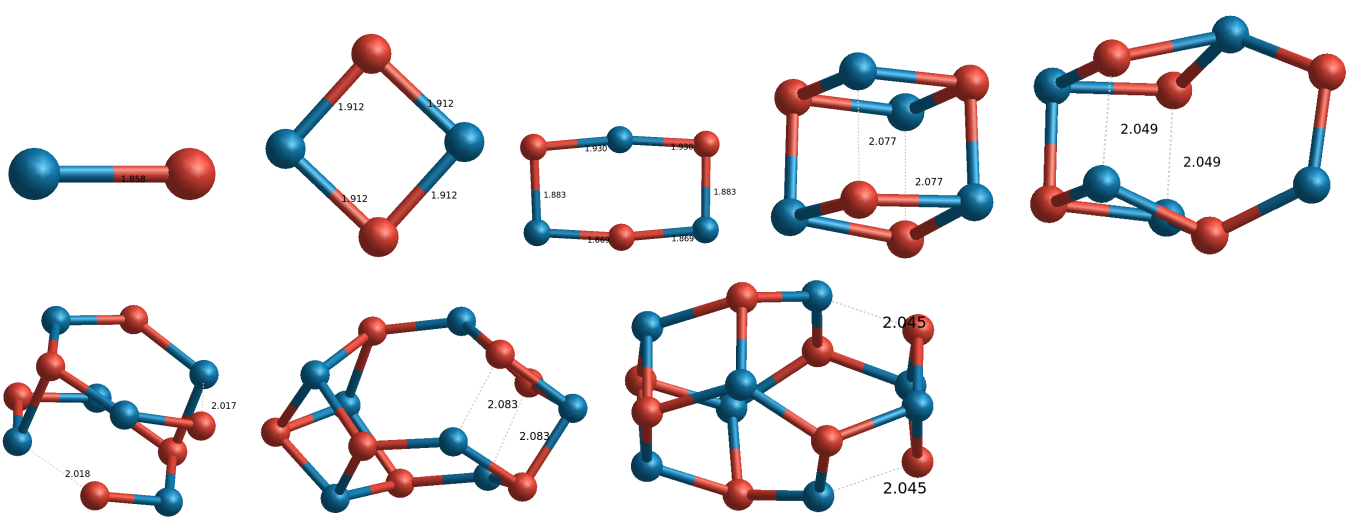

Figure 2. The adiabatically optimized structures of $(\mathrm{MgO})_{\mathrm{n}}{ }^{+}$cations. $\mathrm{Mg}$ atoms are in blue; $\mathrm{O}$ atoms in red; numbers correspond to bond distances in $\AA$.

\subsubsection{Silicon Monoxide Cations}

The Si-O bond length of the $\mathrm{SiO}^{+}$cation is only marginally larger $(<0.01 \AA)$ than that of the neutral $\mathrm{SiO}$ molecule (see Figure 3 ). It is thus not surprising that vertical and adiabatic ionization energies have the same value $(11.49 \mathrm{eV})$. This ionization energy is the largest in our sample and reflects the particular stability of the $\mathrm{SiO}$ molecule. The $\mathrm{SiO}$ dimer, $(\mathrm{SiO})_{2}$, and its cation, $(\mathrm{SiO})_{2}{ }^{+}$, both have an ideal rhombic conformation with identical edge lengths. As cations, they are slightly shorter $(1.699 \AA)$ than as neutral clusters $(1.717 \AA)$. This difference accounts for just $0.01 \mathrm{eV}$ of the large ionization energy of $9.21 \mathrm{eV}$. In the $(\mathrm{SiO})_{3}{ }^{+}$cation, two long $(1.775 \AA)$ and two short $(1.674 \AA) \mathrm{Si}-\mathrm{O}$ bonds appear, whereas they are all identical in the neutral $(\mathrm{SiO})_{3}$ cluster $(1.691 \AA)$. This structural rearrangement lowers the energy by $0.27 \mathrm{eV}$. Both neutral and cationic $(\mathrm{SiO})_{4}{ }^{(+)}$, have identical lengths for all bonds. Their difference is very small $(0.003 \AA)$. Additionally, the bond angles of this non-planar geometry are hardly different $\left(<4^{\circ}\right)$, which is reflected in the small $\mathrm{E}^{\mathrm{v}}-\mathrm{E}^{\mathrm{a}}$ difference of $0.05 \mathrm{eV}$. The majority of the $\mathrm{Si}-\mathrm{O}$ bonds in $(\mathrm{SiO})_{5}{ }^{+}$(i.e., where the lengths are indicated) display ionization-induced changes in their length by $0.05-0.07 \AA$, leading to a significant reduction in the energy $(0.54 \mathrm{eV})$. The remaining three $\mathrm{Si}-\mathrm{O}$ bonds are almost identical in the neutral and cationic forms. At this size $(n=5)$ the adiabatic ionization energy has dropped below $8 \mathrm{eV}$ and is thus similar to those of the $(\mathrm{MgO})_{\mathrm{n}}$. As for $\mathrm{n}=5$, there are three $\mathrm{Si}-\mathrm{O}$ bonds that hardly change in $(\mathrm{SiO})_{6}$ through ionization. All other bonds (indicated by their bond lengths) deviate about $0.04-0.09 \AA$ as compared to the neutral cluster, resulting in an energy decrease of $0.59 \mathrm{eV}$. For $\mathrm{n}=7$, no large geometric changes were observed in the bond lengths (maximum $0.03 \AA$ for $\mathrm{Si}-\mathrm{O}$ bonds). However, the three Si-Si bonds were shorter by $0.05 \AA$, as compared to the neutral cluster, accounting for about $0.70 \mathrm{eV}$. This size represents an outlier in the trend of decreasing ionization energies with respect to the cluster size $n$.

In $(\mathrm{SiO})_{8}{ }^{+}$, only two bonds (one $\mathrm{Si}-\mathrm{Si}$, one $\mathrm{Si}-\mathrm{O}$, indicated by their bond lengths) were altered significantly in the ionization process, accounting for $0.42 \mathrm{eV}$. 
With the exception of $\mathrm{n}=7,(\mathrm{SiO})_{n}$ clusters showed decreasing vertical and adiabatic ionization energies with increasing cluster size $\mathrm{n}$. Moreover, we found that for the small cation clusters $(n=1-6)$, exhibiting strict cation-anion ordering, the ionization process could not (unambiguously) be attributed to a specific site. For the larger clusters $(\mathrm{n}=7-8)$ the electron removal could be localized towards the silicon segregation of the cluster. Our dataset is too small to draw firm conclusions, but our study indicates that the segregation of silicon impacts the ionization energies, possibly reducing them by supplying sites with smaller charge separation.

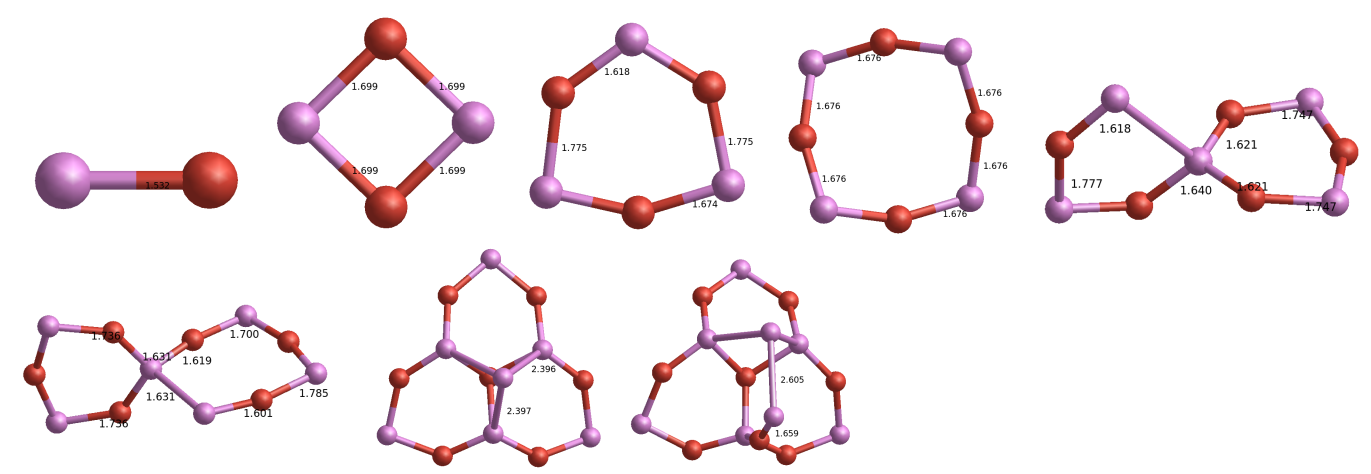

Figure 3. The adiabatically optimized stuctures of $(\mathrm{SiO})_{n}$ cations. $\mathrm{Si}$ atoms are in violet, $\mathrm{O}$ atoms in red, numbers correspond to bond distances in $\AA$.

\subsubsection{Alumina Cations}

The neutral $\mathrm{Al}_{2} \mathrm{O}_{3}$ monomer is a triplet state with a "kite"-shaped geometry. In contrast to the neutral cluster with quite similar Al-O bond lengths (1.747-1.777 $\AA$ ), the $\left(\mathrm{Al}_{2} \mathrm{O}_{3}\right)^{+}$cation exhibits two longer bonds $(1.817 \AA)$ and two shorter bonds $(1.696 \AA)$, as can be seen in Figure 4. The removed electron thus originates potentially from the terminal $\mathrm{Al}$ atom, leaving another unpaired electron at the opposite site of the cluster (i.e., the tail of the kite). The monomer has an adiabatic ionization energy of $9.15 \mathrm{eV}$, which is lower than for larger alumina clusters (with the exception of $n=8$ ). The adiabatic ionization of the highly symmetric neutral alumina dimer (point group $\mathrm{T}_{\mathrm{d}}$ ) leads to a TS. By distorting the TS geometry, we found a true $\left(\mathrm{Al}_{2} \mathrm{O}_{3}\right)_{2}{ }^{+}$minimum showing bonds with pairwise identical lengths and a corresponding lower symmetry (point group $\mathrm{C}_{2}$ ). The energy difference between the TS and the real cation minimum is just $0.03 \mathrm{eV}$. For $n=3$, the largest change, induced by the adiabatic ionization, comes from a newly arising bond with a length of $1.968 \AA$ that is not present in the neutral cluster (distance $2.279 \AA$ ). As a consequence of this new bond, the difference between vertical and adiabtic ionization $(0.53 \mathrm{eV})$ is the largest among all considered alumina clusters. The alumina tetramer cation shows four bonds that are enlarged by $0.07-0.10 \AA$ (indicated by their bond lengths) as compared to the neutral $\left(\mathrm{Al}_{2} \mathrm{O}_{3}\right)_{4}$ cluster. The adiabatic and vertical ionization energies differ by $0.36 \mathrm{eV}$ and account for $9.52 \mathrm{eV}$ and $9.88 \mathrm{eV}$, respectively. In $\left(\mathrm{Al}_{2} \mathrm{O}_{3}\right)_{5}{ }^{+}$, three $\mathrm{Al}-\mathrm{O}$ bonds (lengths are given in Figure 4) are predominantly affected by the ionization process in changing their magnitude by $0.07-0.09 \AA$. The adiabatic ionization of $\left(\mathrm{Al}_{2} \mathrm{O}_{3}\right)_{6}{ }^{+}$does not change the cluster geometry significantly, which is reflected by moderate adaptations in the bond lengths (maximum $0.04 \AA$ ) and energies $(0.19 \mathrm{eV})$. Moreover, the $\mathrm{C}_{2 \mathrm{~h}}$ symmetry of the cluster is preserved. For $n=7$, the changes in the cluster geometry could be localized at one site (see bond length tags in Figure 4) and account for changes of $0.08-0.17 \AA$. The difference between adiabatic and vertical ionization energy is $0.24 \mathrm{eV}$. The geometry of the $\left(\mathrm{Al}_{2} \mathrm{O}_{3}\right)_{8}{ }^{+}$cation resembles closely that of the neutral cluster. Two Al-O bonds deviate by $0.05 \AA$ (indicated by numbers). All other bonds change much less. The $C_{2}$ symmetry is preserved.

For alumina clusters of size $n=2-7$, the ionization energies are in a very narrow range of 9.45-9.54 eV, but are smaller for $\mathrm{n}=1(9.15 \mathrm{eV})$ and $\mathrm{n}=8(8.90 \mathrm{eV})$. The comparatively low ionization potential of the monomer $(n=1)$ can be explained by the disposal of an 
unpaired electron that does not constitute a bond. For $\mathrm{n}=8$, we do not have a concise rationale, but it could be a size effect. All considered clusters families show lower energies for $\mathrm{n}=8$ than for smaller sizes $(\mathrm{n}=1-7)$.
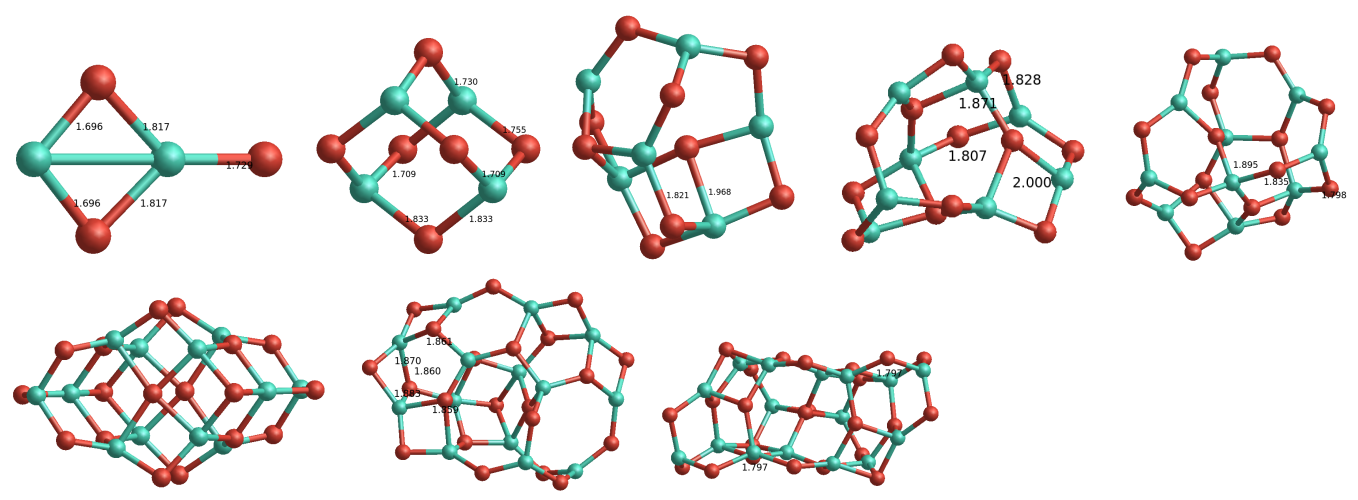

Figure 4. The adiabatically optimized structures of $\left(\mathrm{Al}_{2} \mathrm{O}_{3}\right)_{\mathrm{n}}{ }^{+}$cations. $\mathrm{Al}$ atoms are in green; $\mathrm{O}$ atoms in red; numbers correspond to bond distances in $\AA$.

\subsubsection{Titania Cations}

The adiabatic ionization of the $\mathrm{TiO}_{2}$ molecule results in a TS exhibiting an imaginary vibrational frequency. By stretching one of the two Ti-O bonds, we found a real $\mathrm{TiO}_{2}{ }^{+}$ minimum with two different bond lengths (1.678 $\AA$ and $1.622 \AA$; see Figure 5), as opposed to the neutral $\mathrm{TiO}_{2}$ molecule, which shows two symmetric bonds with a length of $1.64 \AA$. We note a smaller bond angle of $95.2^{\circ}$ as compared to the neutral counterpart $\left(111.7^{\circ}\right)$. The energy difference from the adiabatic optimization is $0.14 \mathrm{eV}$, and the TS energy is less than $0.001 \mathrm{eV}$ above the real minimum. The titania dimer $(n=2)$ hardly changes its geometry when it is adiabatically ionized and preserves its $C_{2 h}$ symmetry. All six bonds change by less than $0.02 \AA$, accounting for $0.27 \mathrm{eV}$. For $\left(\mathrm{TiO}_{2}\right)_{3}$, the overall geometry is not largely affected, since the cluster coordinates change by a maximum of $0.06 \AA$ through adiabatic ionization. The structure of the titania tetramer $(n=4)$ cation is virtually not altered by the ionization process (max. $0.01 \AA$ ), which is reflected in a moderate energy change $(0.11 \mathrm{eV})$. The $\left(\mathrm{TiO}_{2}\right)_{5}{ }^{+}$cation differs from the neutral $\left(\mathrm{TiO}_{2}\right)_{5}$ mostly by one Ti atom (marked in yellow), whose four Ti-O bonds change by $0.08-0.21 \AA$ and are responsible for the energy difference of $0.93 \mathrm{eV}$. In $\left(\mathrm{TiO}_{2}\right)_{6}{ }^{+}$, one $\mathrm{Ti}$ atom (marked in yellow), which is located next to a terminal $\mathrm{O}$ atom, is most strongly affected by the adiabatic ionization accounting for almost $1 \mathrm{eV}$. This Ti atom shows bond lengths $(\Delta \mathrm{d}=0.09-0.21 \AA)$ that differ from those of the neutral cluster. $\left(\mathrm{TiO}_{2}\right)_{7}{ }^{+}$exhibits the most significant changes at one $\mathrm{Ti}$ atom (marked in yellow) that is connected to a terminal, single-bonded $\mathrm{O}$ atom. Here, the bond lengths change due to the ionization by $0.12-0.21 \AA$ and the energy by $0.13 \mathrm{eV}$. For $\mathrm{n}=8$, the adiabatic ionization of the neutral GM candidate did not converge. Even the vertical ionization energy could not be determined. Therefore, we performed ionization calculations for a number of low-energy $\left(\mathrm{TiO}_{2}\right)_{8}$ isomers. In the case of convergence, their adiabatic ionization energies were in the range of 8.76-9.21 eV. As a reference, we used the neutral $\left(\mathrm{TiO}_{2}\right)_{8}$ isomer with the lowest relative energy $(0.26 \mathrm{eV})$ with respect to the GM candidate. Its adiabatic ionization energy was $9.04 \mathrm{eV}$ and was thus the lowest among the titania clusters. The largest geometric change was observed at one Ti atom, whose coordinates changed by $0.11-0.22 \AA$ (bond lengths are indicated), and they had considerably lower energy (by $1.04 \mathrm{eV}$ ).

The titania clusters showed large adiabatic ionization energies ranging from 9.04-10.43 eV. Additionally, the differences between vertical and adiabatic energies were non-negligible. They exhibited an alternating pattern in their ionization energies. Clusters with even size $(n=2,4,6)$ showed higher energies than their neighboring sizes with odd numbers $(n=1,3$, $5,7)$. 


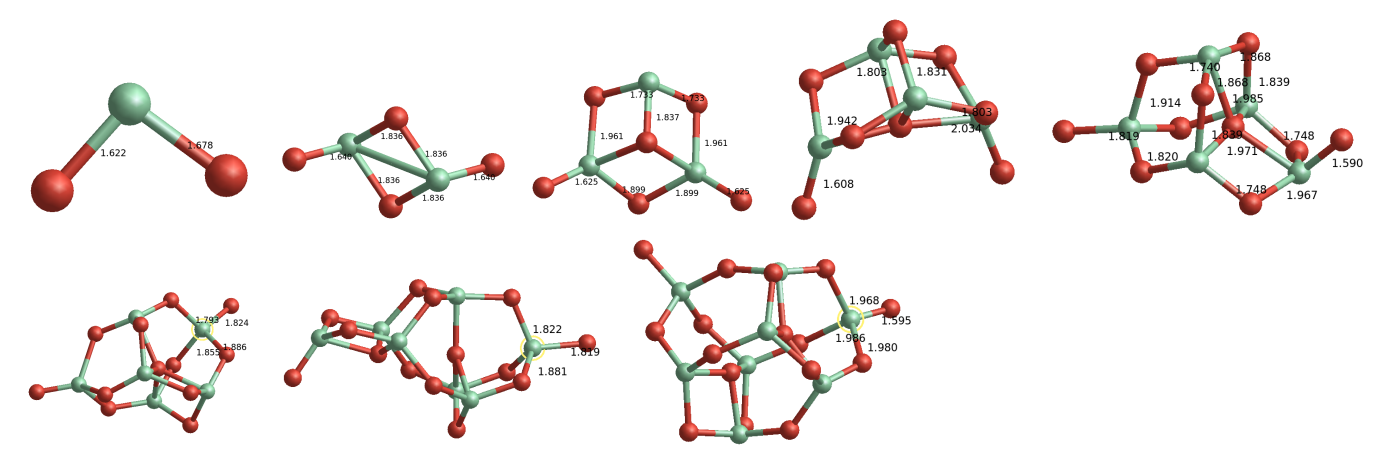

Figure 5. The adiabatically optimized structures of $\left(\mathrm{TiO}_{2}\right)_{\mathrm{n}}$ cations. $\mathrm{Ti}$ atoms are in olive; $\mathrm{O}$ atoms in red; numbers correspond to bond distances in $\AA$.

\section{Discussion and Conclusions}

Dust-forming metal-oxide clusters show ionization energies lower than that of atomic oxygen $(13.6 \mathrm{eV})$, but higher or comparable to those of the corresponding metals. $\mathrm{MgO}$ clusters show generally the lowest ionization energies, which lie in a narrow range of 7.09-8.19 eV, overlapping with the atomic $\mathrm{Mg}$ ionization energy of $7.65 \mathrm{eV} . \mathrm{Al}_{2} \mathrm{O}_{3}$ clusters exhibit ionization energies (8.9-10.0 eV) higher than that of atomic $\mathrm{Al}(5.99 \mathrm{eV})$, with the triplet monomer representing a special case. $\mathrm{TiO}_{2}$ clusters have the highest ionization energies (9.27-10.54 eV) among the considered metal oxides, exceeding the atomic Ti ionization potential of $6.83 \mathrm{eV}$. SiO clusters have the largest variation in their ionization energies (6.62-11.49 eV), which monotonically decrease with size, thereby crossing the atomic $\mathrm{Si}$ ionization energy of $8.15 \mathrm{eV}$. The decrease might be the result of the size-dependent atomic segregation of $(\mathrm{SiO})_{n}$ clusters. All considered cluster families have a gradually declining trend of ionization energy with cluster size.

Gas constituents usually do not have a single temperature, but follow a statistical distribution function. Assuming chemical equilibrium, we can assess the fraction of ionized clusters by using the Boltzmann law:

$$
\frac{n\left(X^{+}\right)}{n(X)}=\frac{2}{1} \exp \left(-\frac{E_{i}}{K T}\right)
$$

where $n\left(X^{+}\right)$and $n(X)$ are the number densities of the ionized and neutral cluster species $X$, respectively. $E_{i}$ is the previously introduced ionization energy and $K T$ corresponds to the thermal energy. The factor $\frac{2}{1}$ arises from the spin multiplicity $\mathrm{M}$ of the neutrals $(\mathrm{M}=1)$ and cations $(\mathrm{M}=2)$ and accounts for the statistical weights. Note that for the triplet $\mathrm{X}=\mathrm{Al}_{2} \mathrm{O}_{3}$, it should read $\frac{2}{3}$.

From Table 1 it is apparent that the ionization energies range from 6.62 to $11.49 \mathrm{eV}$, corresponding to equivalent kinetic temperatures of 76,800-133,300 K. These temperatures are orders of magnitude larger than the prevailing photospheric temperatures of an AGB star, which are typically between 2000 and $3000 \mathrm{~K}$. According to Equation (2), the ionization fractions at the photosphere result in small values between $2.3 \times 10^{-29}$ and $1.5 \times 10^{-11}$ for $\mathrm{T} \simeq 2000-3000 \mathrm{~K}$. However, pulsation-induced shocks, traveling periodically through the circumstellar envelopes of AGB stars, increase the temperature locally and temporarily. By applying the Rankine-Hugoniot jump conditions to photospheric conditions and a diatomic gas, temperatures of $\sim 40,000 \mathrm{~K}$ can be attained (see e.g., [39]). This is still a factor of 2-3 smaller than the equivalent ionization temperatures. However, owing to its intrinsic chemical equilibrium assumption, Equation (2) is not applicable to an immediate post-shock gas strongly deviating from equilibrium conditions.

Finally, we want to address radiation fields. Stellar radiation fields from AGB stars with temperatures of 2000-3000 K typically peak at micrometer wavelengths in the infrared. Though the majority of the stellar photons are thus not capable of ionizing the presented metal oxide clusters, there is a certain probability that UV photons originating from the high energy tail of their distribution induce ionization. Although the ionization fraction 
is comparatively low at characteristic AGB temperatures, the orders-of-magnitude faster ion-molecule chemistry with rates up to $10^{0} \mathrm{~cm}^{3} \mathrm{~s}^{-1}$ could compete with neutral-neutral reaction rates that usually range between $10^{-9}$ and $10^{-13} \mathrm{~cm}^{3} \mathrm{~s}^{-1}$. While accounting for both the fast kinetic rates (i.e., $9-13$ orders of magnitude) and the low abundances of the cluster cations (i.e., 11-29 orders of magnitude), we found that the ion-molecule chemistry can compete with neutral-neutral reactions for the lowest ionization energies $(<8 \mathrm{eV})$ and the fastest ion-molecule rates. Moreover, pulsation-induced shocks are prone to increase the amount of ions and therefore also enhance their impact on the circumstellar chemistry. Therefore, an ion-molecule driven chemistry can become important in the dust formation zone of AGB stars, particularly in the immediate post-shock regime. However, neutralneutral reactions are still believed to represent the dominant nucleation mechanism, as only extreme cases (i.e., reactions with the fastest ion-molecule rates and the largest ion concentrations corresponding to low ionization energies) occur on comparable timescales. We conclude that albeit ionization of the metal oxide clusters is of secondary importance under equilibrium conditions, non-equilibrium effects, such as periodic shocks or radiation from a hot companion star, can provide the energy required to ionize (some of) the metal oxide clusters. Moreover, the (inter)stellar radiation field is a promising source of photons with sufficiently large energies capable of ionizing metal oxide clusters. [40] investigated the effect of stellar UV photons on the chemistry occurring in AGB winds and found that photo-dissociation can considerably change the related molecular abundances. It is thus possible that photo-ionization also takes place. The presence of metal-oxide cations would impart significant effects on the nucleation pathways, their related energies, and their growth rates. A realistic description of the temperature structure and radiation field in the highly dynamic envelopes of AGB stars is, however, beyond the scope of this paper.

Author Contributions: Conceptualization, D.G.; validation, J.P.S., H.L.-M., L.D.; investigation, D.G., J.P.S.; data curation, D.G.; writing — original draft preparation, D.G.; writing-review and editing, J.P.S., H.L.-M., L.D.; funding acquisition, L.D. All authors have read and agreed to the published version of the manuscript.

Funding: D.G. and L.D. acknowledge funding by the ERC consolidator grant number 646758. J.P.S. and H.L.-M. acknowledge the European Union's Horizon 2020 research and innovation program under the Marie Sklodowska-Curie grant, agreement number 860470.

Institutional Review Board Statement: Not applicable.

Informed Consent Statement: Not applicable.

Data Availability Statement: Data are contained within the article. The data presented in this study are available at http:/ / dave202.bplaced.net/ions/, accessed on 1 March 2021.

Acknowledgments: We acknowledge the CINECA award under the ISCRA initiative, for the availability of high performance computing resources and support.

Conflicts of Interest: The authors declare no conflict of interest.

\section{References}

1. Höfner, S.; Olofsson, H. Mass loss of stars on the asymptotic giant branch. Mechanisms, models and measurements. Astron. Astrophys. Rev. 2018, 26. [CrossRef]

2. Henning, T.; Begemann, B.; Mutschke, H.; Dorschner, J. Optical properties of oxide dust grains. Astron. Astrophys. Suppl. Ser. 1995, 112, 143.

3. Zinner, E.K. Presolar Grains. Treatise Geochem. 2003, 1, 711. [CrossRef]

4. Nittler, L.R.; Alexander, C.M.O.; Stroud, R.M. High Abundance of Presolar Materials in CO3.0 Chondrite Dominion Range 08006. In Proceedings of the Lunar and Planetary Science Conference, The Woodlands, TX, USA, 18-22 March 2013 ; p. 2367.

5. Hoppe, P.; Leitner, J.; Kodolányi, J. New Constraints on the Abundances of Silicate And Oxide Stardust from Supernovae in the Acfer 094 Meteorite. Astrophys. J. 2015, 808, L9. [CrossRef]

6. Jaeger, C.; Molster, F.J.; Dorschner, J.; Henning, T.; Mutschke, H.; Waters, L.B.F.M. Steps toward interstellar silicate mineralogy. IV. The crystalline revolution. Astron. Astrophys. 1998, 339, 904-916.

7. Min, M.; Waters, L.B.F.M.; de Koter, A.; Hovenier, J.W.; Keller, L.P.; Markwick-Kemper, F. The shape and composition of interstellar silicate grains. Astron. Astrophys. 2007, 462, 667-676. [CrossRef] 
8. $\quad$ Kemper, F.; Waters, L.B.F.M.; de Koter, A.; Tielens, A.G.G.M. Crystallinity versus mass-loss rate in asymptotic giant branch stars. Astron. Astrophys. 2001, 369, 132-141. [CrossRef]

9. Goumans, T.P.M.; Bromley, S.T. Efficient nucleation of stardust silicates via heteromolecular homogeneous condensation. Mon. Not. R. Astron. Soc. 2012, 420, 3344-3349. [CrossRef]

10. Escatllar, A.M.; Lazaukas, T.; Woodley, S.M.; Bromley, S.T. Structure and Properties of Nanosilicates with Olivine (Mg2SiO4)N and Pyroxene $\left(\mathrm{MgSiO}_{3}\right) \mathrm{N}$ Compositions. ACS Earth Space Chem. 2019, 3, 2390-2403. [CrossRef]

11. Ferguson, F.T.; Nuth, J.A. Vapor Pressure of Silicon Monoxide. J. Chem. Eng. Data 2008, 53, 2824-2832. [CrossRef]

12. Gail, H.P.; Wetzel, S.; Pucci, A.; Tamanai, A. Seed particle formation for silicate dust condensation by SiO nucleation. Astron. Astrophys. 2013, 555, A119, [CrossRef]

13. Norris, B.R.M.; Tuthill, P.G.; Ireland, M.J.; Lacour, S.; Zijlstra, A.A.; Lykou, F.; Evans, T.M.; Stewart, P.; Bedding, T.R. A close halo of large transparent grains around extreme red giant stars. Nature 2012, 484, 220-222. [CrossRef]

14. Höfner, S.; Gautschy-Loidl, R.; Aringer, B.; Jørgensen, U.G. Dynamic model atmospheres of AGB stars. III. Effects of frequencydependent radiative transfer. Astron. Astrophys. 2003, 399, 589-601. [CrossRef]

15. Boulangier, J.; Gobrecht, D.; Decin, L.; de Koter, A.; Yates, J. Developing a self-consistent AGB wind model: II. Non-classical, non-equilibrium polymer nucleation in a chemical mixture. Mon. Not. R. Astron. Soc. 2019, [CrossRef]

16. Posch, T.; Kerschbaum, F.; Mutschke, H.; Fabian, D.; Dorschner, J.; Hron, J. On the origin of the 13 mu m feature. A study of ISO-SWS spectra of oxygen-rich AGB stars. Astron. Astrophys. 1999, 352, 609-618.

17. Posch, T.; Kerschbaum, F.; Fabian, D.; Mutschke, H.; Dorschner, J.; Tamanai, A.; Henning, T. Infrared Properties of Solid Titanium Oxides: Exploring Potential Primary Dust Condensates. Astrophys. J. Suppl. Ser. 2003, 149, 437-445. [CrossRef]

18. Sloan, G.C.; Kraemer, K.E.; Goebel, J.H.; Price, S.D. Guilt by Association: The 13 Micron Dust Emission Feature and Its Correlation to Other Gas and Dust Features. Astrophys. J. 2003, 594, 483-495. [CrossRef]

19. Koehler, T.M.; Gail, H.P.; Sedlmayr, E. MgO dust nucleation in M-Stars: Calculation of cluster properties and nucleation rates. Astron. Astrophys. 1997, 320, 553-567.

20. Bromley, S.T.; Gomez Martin, J.C.; Plane, J.M.C. Under what conditions does (SiO)N nucleation occur? A bottom-up kinetic modelling evaluation. Phys. Chem. Chem. Phys. 2016, 18, 26913-26922. [CrossRef] [PubMed]

21. Decin, L.; Richards, A.M.S.; Waters, L.B.F.M.; Danilovich, T.; Gobrecht, D.; Khouri, T.; Homan, W.; Bakker, J.M.; Van de Sande, M.; Nuth, J.A.; et al. Study of the aluminium content in AGB winds using ALMA - Indications for the presence of gas-phase $\left(\mathrm{Al}_{2} \mathrm{O}_{3}\right)$ n clusters. Astron. Astrophys. 2017, 608, A55. [CrossRef]

22. Demyk, K.; van Heijnsbergen, D.; von Helden, G.; Meijer, G. Experimental study of gas phase titanium and aluminum oxide clusters. Astron. Astrophys. 2004, 420, 547-552. [CrossRef]

23. Haertelt, M.; Fielicke, A.; Meijer, G.; Kwapien, K.; Sierka, M.; Sauer, J. Structure determination of neutral MgO clusters-hexagonal nanotubes and cages. PCCP 2012, 14, 2849-2856. [CrossRef] [PubMed]

24. Savoca, M.; Langer, J.; Harding, D.J.; Palagin, D.; Reuter, K.; Dopfer, O.; Fielicke, A. Vibrational spectra and structures of bare and Xe-tagged cationic SinOm+ clusters. J. Chem. Phys. 2014, 141, 104313. [CrossRef]

25. Bertschinger, E.; Chevalier, R.A. A periodic shock wave model for Mira variable atmospheres. Astrophys. J. 1985, 299, 167-190. [CrossRef]

26. Mathis, J.S.; Mezger, P.G.; Panagia, N. Interstellar radiation field and dust temperatures in the diffuse interstellar matter and in giant molecular clouds. Astron. Astrophys. 1983, 500, 259-276.

27. Becke, A.D. A new mixing of Hartree-Fock and local density-functional theories. J. Chem. Phys. 1993, 98, 1372-1377. [CrossRef]

28. Corà, F. The performance of hybrid density functionals in solid state chemistry: The case of $\mathrm{BaTiO}_{3}$. Mol. Phys. 2005, 103, 2483-2496. [CrossRef]

29. Frisch, M.J.; Trucks, G.W.; Schlegel, H.B.; Scuseria, G.E.; Robb, M.A.; Cheeseman, J.R.; Scalmani, G.; Barone, V.; Mennucci, B.; Petersson, G.A.; et al. Gaussian 09 Revision A.1, 2010; Gaussian Inc.: Wallingford, UK, 2009.

30. Chen, M.; Felmy, A.R.; Dixon, D.A. Structures and Stabilities of (MgO)n Nanoclusters. J. Phys. Chem. A 2014, 118, 3136-3146. [CrossRef]

31. Li, R.; Cheng, L. Structural determination of $\left(\mathrm{Al}_{2} \mathrm{O}_{3}\right) \mathrm{n}(\mathrm{n}=1-7)$ clusters based on density functional calculation. Comput. Theor. Chem. 2012, 996, 125-131. [CrossRef]

32. Gobrecht, D.; Decin, L.; Cristallo, S.; Bromley, S.T. A global optimisation study of the low-lying isomers of the alumina octomer $\left(\mathrm{Al}_{2} \mathrm{O}_{3}\right) 8$. Chem. Phys. Lett. 2018, 711, 138-147. [CrossRef]

33. Lamiel-Garcia, O.; Cuko, A.; Calatayud, M.; Illas, F.; Bromley, S.T. Predicting size-dependent emergence of crystallinity in nanomaterials: Titania nanoclusters versus nanocrystals. Nanoscale 2017, 9, 1049-1058. [CrossRef] [PubMed]

34. Dalleska, N.; Armentrout, P. Guided ion beam studies of reactions of alkaline earth ions with $\mathrm{O}_{2}$. Int. J. Mass Spectrom. Ion Process. 1994, 134, 203-212. [CrossRef]

35. Lemmon, E.W.; McLinden, M.O.; Friend, D.G. Thermophysical properties of fluid systems. In NIST Chemistry Webbook, NIST Standard Reference Database; Linstrom, P.J., Mallard, W.G., Eds.; National Institute of Standards and Technology: Gaithersburg, MD, USA, 1998; Volume 69.

36. Armentrout, P.B.; Halle, L.F.; Beauchamp, J.L. Reaction of $\mathrm{Cr}+, \mathrm{Mn}+, \mathrm{Fe}+, \mathrm{Co}+$, and $\mathrm{Ni}+$ with $\mathrm{O}_{2}$ and $\mathrm{N}_{2} \mathrm{O}$. Examination of the translational energy dependence of the cross sections of endothermic reactions. J. Chem. Phys. 1982, 76, 2449-2457. [CrossRef] 
37. Terzi, N.; Yaghlane, S.B.; Jaïdane, N.E.; Chambaud, G.; Hochlaf, M. Neutral and Multicharged Ions of Small Aluminum Oxides: Structures, Spectroscopy, and Energetics. J. Phys. Chem. A 2020, 124, 9021-9034. [CrossRef]

38. Dehnicke, K.; Weidlein, J. Existence of the $\mathrm{VO}, \mathrm{TiO}^{2+}$ and $\mathrm{ZrO}^{2+}$ Cations. Angew. Chem. Int. Ed. Engl. 1966, 5, 1041-1041. [CrossRef]

39. Gobrecht, D.; Cherchneff, I.; Sarangi, A.; Plane, J.M.C.; Bromley, S.T. Dust formation in the oxygen-rich AGB star IK Tauri. Astron. Astrophys. 2016, 585, A6. [CrossRef]

40. Van de Sande, M.; Millar, T.J. The Role of Internal Photons on the Chemistry of the Circumstellar Envelopes of AGB Stars. Astrophys. J. 2019, 873, 36. [CrossRef] 